\title{
Characteristics of elderly patients with polypharmacy who refuse to participate in an in-hospital deprescribing intervention: a retrospective cross-sectional study
}

\author{
Junpei Komagamine ${ }^{1^{*}} \mathbb{D}$, Kenichi Sugawara ${ }^{2}$ and Kazuhiko Hagane ${ }^{3}$
}

\begin{abstract}
Background: Few studies have evaluated the characteristics of elderly patients with polypharmacy refusing deprescribing. The aim of this study was to evaluate the prevalence of potentially inappropriate medication (PIM) use in elderly patients accepting and refusing a deprescribing intervention and to investigate factors associated with deprescribing refusal.

Methods: We conducted a retrospective cross-sectional study by analyzing the electronic medical records from a single hospital. All consecutive patients aged 65 years or older who reported the use of five or more medications upon admission to the orthopedic ward from January 2015 to December 2016 and who were approached by a pharmacist for polypharmacy screening were included. Patients who had provided consent for the deprescribing intervention by the internal medicine physicians were defined as the acceptance group, and patients who did not were defined as the refusal group. The primary outcome was the use of any PIMs at admission, based on the 2015 American Geriatric Society Beers Criteria. Using multivariable logistic regression, predictive factors of refusing deprescribing were also evaluated.
\end{abstract}

Results: During the study period, 136 patients were eligible. Of those, 82 patients (60.3\%) accepted the deprescribing intervention, and 54 patients (39.7\%) declined the intervention. The mean age of all the patients was 81.1 years, and the mean number of medications at admission was 9.3. The overall proportion of patients taking any PIMs at admission was $77.2 \%$. The proportion of patients taking any PIMs at admission was not different between the acceptance and refusal groups ( $78.0 \%$ and $75.9 \%$, respectively; $p=0.84$ ). None of the measured characteristics, including age, gender, residential status, comorbidity, alcohol use, smoking status, number of medications, or number of PIMs, were found to be associated with deprescribing refusal.

Conclusion: The prevalence of any PIM use did not differ among elderly orthopedic patients with polypharmacy according to refusal or acceptance of the deprescribing intervention. Furthermore, none of the analyzed characteristics were found to be associated with deprescribing refusal. Given the high prevalence of PIM use, a strategy is needed for combating polypharmacy among elderly patients reluctant to undergo deprescribing.

Keywords: Deprescribing, Polypharmacy, Potentially inappropriate medications

\footnotetext{
* Correspondence: junpei0919@yahoo.co.jp

${ }^{1}$ Department of Internal Medicine, National Hospital Organization Tochigi

Medical Center, 1-10-37, Nakatomatsuri, Utsunomiya, Tochigi 3208580, Japan

Full list of author information is available at the end of the article
}

(c) The Author(s). 2018 Open Access This article is distributed under the terms of the Creative Commons Attribution 4.0 International License (http://creativecommons.org/licenses/by/4.0/), which permits unrestricted use, distribution, and reproduction in any medium, provided you give appropriate credit to the original author(s) and the source, provide a link to the Creative Commons license, and indicate if changes were made. The Creative Commons Public Domain Dedication waiver (http://creativecommons.org/publicdomain/zero/1.0/) applies to the data made available in this article, unless otherwise stated. 


\section{Background}

Polypharmacy, a common problem among elderly patients due to multi-morbidities [1,2], is associated with inappropriate prescribing [3], which can be harmful [4]. Recently, deprescribing has been suggested to reduce inappropriate polypharmacy among elderly patients [5-7]. Deprescribing is a systematic process of identifying and discontinuing drugs in instances in which existing or potential harms outweigh existing or potential benefits within the context of an individual patient's care goals, current level of functioning, life expectancy, values, and preferences [7]. Although it is uncertain whether deprescribing in elderly patients improves clinical outcomes, this strategy can safely and effectively reduce the use of potentially inappropriate medications (PIMs) [8-10].

Nonetheless, there are some barriers for deprescribing in elderly patients with inappropriate polypharmacy. These barriers result from patient-, prescriber- and system-related factors [11-13]. Patient factors include fear of consequences of cessation and feeling that a medication is currently beneficial for a condition. Past randomized controlled studies for deprescribing in elderly patients with polypharmacy have reported that over $30 \%$ of eligible patients refused to undergo deprescribing and were thus excluded from the study [14-16]. This refusal rate seems overly high given that other recent studies evaluating elderly peoples' attitudes regarding polypharmacy reported that $80-92 \%$ of participants are willing to have one or more medications deprescribed if their doctor said it was possible [17-19]. This gap might reflect the difference between real-world practice and survey studies. Even patients who indicate they prefer deprescribing in a survey might refuse when actually recommended to stop their medications. However, these studies lacked information regarding the appropriateness of medications among patients who refused deprescribing; this lack of information is problematic if the PIM prevalence for polypharmacy patients who refuse deprescribing is similar to or higher than the PIM prevalence for those who accept deprescribing. To our knowledge, no studies have evaluated the difference in PIM prevalence in elderly patients with polypharmacy who refuse or accept deprescribing. Furthermore, factors associated with deprescribing refusal in elderly patients with polypharmacy have not been evaluated in a real-world practice. Thus, our aim was to evaluate PIM prevalence in elderly patients with polypharmacy who accept or refuse deprescribing as well as factors associated with deprescribing refusal.

\section{Methods}

\section{Study design and participants}

A retrospective cross-sectional study was conducted using the database of the National Hospital Organization
Tochigi Medical Center, with data collected from January 1, 2015 to December 31, 2016. The National Hospital Organization Tochigi Medical Center is a 350bed acute care hospital in the Tochigi prefecture of Japan. All consecutive patients aged 65 years or more with 5 or more medications at admission who were admitted to the orthopedic ward in our hospital and who were approached by a pharmacist for polypharmacy screening were included. Patients admitted to other wards or aged less than 65 years were excluded. We also excluded patients with a second admission to the orthopedic ward during the study period. The requirement for individual informed consent was formally waived by the Medical Ethical Committee of National Hospital Organization Tochigi Medical Center because the data were collected from medical records and the patients were not contacted. The study protocol was approved by the institutional research ethics committee of National Hospital Organization Tochigi Medical Center (No. 28-20) and was carried out in accordance with the Declaration of Helsinki.

Beginning in January 2015, our hospital started screening and intervening to reduce inappropriate or unnecessary medication use for elderly patients hospitalized in the orthopedic ward. Pharmacists attempted to contact elderly patients prescribed five or more medications at admission and their families to inform them of the intervention to reduce inappropriate or unnecessary medication use. The patients the pharmacists could contact and who provided written consent to receive the polypharmacy intervention were scheduled for an appointment with an internal medicine physician as soon as possible. The physicians took a history from the patients, performed a physical and neurological examination, evaluated the appropriateness of the polypharmacy, changed medications as appropriate and followed up with patients after the consultation until they were discharged. Thus, patients who had given written consent to receive the intervention were defined as the acceptance group, whereas those who did not were defined as the refusal group. This deprescribing intervention was part of the usual care regimen in our hospital rather than a separate research study. However, we did obtain written consent from patients to conduct the intervention as part of the usual care regimen because this practice is unfamiliar in Japan.

\section{Measurements \\ Characteristics}

Information on age, gender, primary diagnosis for admission, social history, past medical history, Charlson Comorbidity Index (CCI) [20], and medications was retrieved from medical records taken at the time of each patient's first admission. Medications were determined based on a comprehensive medication history performed by a pharmacist. Medications included oral medications, 
inhalers, and injections. As-needed medications were also included, but eye drops, intranasal infusers, overthe-counter (OTC) drugs, and topical medications were excluded because the deprescribing intervention of our hospital did not target these medications.

\section{Outcome measures}

The primary outcome was the use of any PIMs at admission. PIMs were defined based on the 2015 Beers Criteria of the American Geriatric Society (AGS) [21]. Of the five parts of the Beers Criteria, we used only two parts: PIM use in older adults and PIM use in older adults due to drug-disease or drug-syndrome interactions that may exacerbate the disease or syndrome. The secondary outcome was the number of total medications and PIMs at admission.

\section{Statistical analysis}

We estimated that a sample of 132 patients would provide the study with a power of at least $80 \%$ to show an absolute difference of $25 \%$ for the primary outcome between the acceptance and refusal groups, assuming that approximately half of eligible patients would decline the intervention (based on previous studies [14-16]) and that the proportion of patients taking any PIMs was 50\% (based on previous studies $[22,23])$ in the acceptance group.

The baseline characteristics of the study population were compared using Fisher's exact test for categorical variables and Student's t-test for continuous variables. The proportion of patients taking any PIMs at admission among the refusal group was compared with those among the acceptance group using Fisher's exact test. To identify the determinants of refusal for deprescribing, we also conducted multivariable analysis using binary logistic regression to examine the association between select variables and refusal. The following variables were entered in the logistic regression model: age, gender, CCI, residential status, dementia, depression, insomnia, current smoker, regular drinker, number of medications at admission, and number of PIMs at admission. These analyses were carried out using Excel statistical software package version 2.11 (Bellcurve for Excel; Social Survey Research Information Co., Ltd., Tokyo, Japan), and the level of significance was set at $5 \%$.

\section{Results}

We screened 178 orthopedic patients at least 65 years old with 5 or more medications during the study period; 40 were excluded because the pharmacists could not contact them to discuss the polypharmacy intervention, and 2 were excluded because of a second admission during the study period. Thus, a total of 136 patients were included in the final analysis. Among these patients, 54 (39.7\%) refused the deprescribing intervention, and 82 patients $(60.3 \%)$ provided consent for the deprescribing intervention.

The baseline characteristics of the patients in each group are presented in Table 1 . For all 136 patients, the mean age was 81.1 years, $34(25.0 \%)$ were men, the mean CCI was 1.9, 26 (19.1\%) had dementia, 17 (12.5\%) were institutional residents, 10 (7.4\%) were current smokers, and $15(11.0 \%)$ were regular drinkers. The mean number of medications at admission was 9.3. These demographic features were similar between the acceptance and refusal groups; however, the refusal group tended to have a higher proportion of current smokers compared with the acceptance group, although this association was not statistically significant $(p=0.051)$.

Table 2 shows the total number of PIMs and the proportions of patients taking any PIMs at admission in each group according to drug subcategory. For all patients, the mean number of PIMs at admission was 1.5, and the proportion of patients taking any PIMs at

Table 1 Characteristics of the 136 included orthopedic patients

\begin{tabular}{|c|c|c|c|}
\hline & \multicolumn{2}{|c|}{ Deprescribing for polypharmacy } & \multirow[t]{3}{*}{$P$-value } \\
\hline & Acceptance & Refusal & \\
\hline & $N=82$ & $N=54$ & \\
\hline Age, mean $\pm S D$ & $80.3 \pm 7.5$ & $82.3 \pm 7.4$ & 0.14 \\
\hline Men, $n(\%)$ & $22(26.8)$ & $12(22.2)$ & 0.69 \\
\hline Institutional resident, $n$ (\%) & $10(12.2)$ & $7(13.0)$ & 1.00 \\
\hline $\mathrm{CCl}$, mean $\pm \mathrm{SD}$ & $1.9 \pm 1.5$ & $1.7 \pm 1.7$ & 0.50 \\
\hline Current smoker, $n$ (\%) & $3(3.7)$ & $7(13.0)$ & 0.05 \\
\hline Regular drinker, $n$ (\%) & $7(8.5)$ & $8(14.8)$ & 0.28 \\
\hline $\begin{array}{l}\text { Number of medications } \\
\text { at admission, mean } \pm \text { SD }\end{array}$ & $9.6 \pm 3.2$ & $9.0 \pm 2.3$ & 0.22 \\
\hline \multicolumn{4}{|c|}{ Primary reason for admission, $n$ (\%) } \\
\hline Fracture & $56(68.3)$ & $41(75.9)$ & 0.44 \\
\hline Osteoarthritis & $6(7.3)$ & $7(13.1)$ & 0.37 \\
\hline Spinal stenosis & $9(11.0)$ & $3(5.6)$ & 0.36 \\
\hline Other & $11(13.4)$ & $3(5.6)$ & 0.16 \\
\hline \multicolumn{4}{|l|}{ Past medical history, $n$ (\%) } \\
\hline Depression & $6(7.3)$ & $8(14.8)$ & 0.25 \\
\hline Insomnia & $28(34.1)$ & $21(38.9)$ & 0.59 \\
\hline Dementia & $15(18.3)$ & $11(20.4)$ & 0.83 \\
\hline Stroke & $23(28.0)$ & $10(18.5)$ & 0.23 \\
\hline Myocardial infarction & $8(9.8)$ & $6(11.1)$ & 0.78 \\
\hline Heart failure & $8(9.8)$ & $3(5.6)$ & 0.53 \\
\hline Diabetes mellitus & $19(23.2)$ & $11(20.4)$ & 0.83 \\
\hline Asthma or COPD & $10(12.2)$ & $4(7.4)$ & 0.57 \\
\hline Rheumatological disease & $10(12.2)$ & $6(11.1)$ & 1.00 \\
\hline Active cancer & $7(8.5)$ & $2(3.7)$ & 0.20 \\
\hline
\end{tabular}

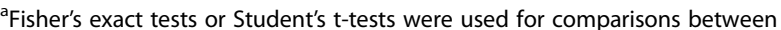
the acceptance and refusal groups. The threshold for statistical significance was set at $p<0.05$ 
Table 2 Prevalence of PIM ${ }^{\mathrm{a}}$ use according to drug subcategory at admission among the 136 included orthopedic patients

\begin{tabular}{llll}
\hline & \multicolumn{2}{l}{$\begin{array}{l}\text { Deprescribing for } \\
\text { polypharmacy }\end{array}$} & \\
\cline { 2 - 3 } & $\begin{array}{llll}\text { Acceptance } \\
\text { Nevelue }\end{array}$ & \\
& $N=82$ & Refusal & \\
\cline { 1 - 2 } Number of PIMs, mean \pm SD & $1.5 \pm 1.2$ & $1.6 \pm 1.3$ & 0.52 \\
Any PIMs, $n$ (\%) & $64(78.0)$ & $41(75.9)$ & 0.84 \\
Category of PIMs, $n$ (\%) & & & \\
Benzodiazepines & $30(36.6)$ & $18(33.3)$ & 0.72 \\
Proton pump inhibitors & $26(31.7)$ & $19(35.2)$ & 0.71 \\
NSAIDs & $7(8.5)$ & $7(13.0)$ & 0.41 \\
Hypnotics & $8(9.8)$ & $5(9.3)$ & 1.00 \\
Anticholinergics & $3(3.7)$ & $6(11.1)$ & 0.16 \\
Anticonvulsive drugs & $4(4.9)$ & $5(9.3)$ & 0.48 \\
Antipsychotics & $7(8.5)$ & $1(1.9)$ & 0.15 \\
Opioids & $2(2.4)$ & $5(9.3)$ & 0.11 \\
Antidepressants & $3(3.7)$ & $4(7.4)$ & 0.43 \\
Ticlopidine or dipyridamole & $5(6.1)$ & $1(1.9)$ & 0.40 \\
Peripheral alpha-1 blocker & $2(2.4)$ & $4(7.4)$ & 0.22 \\
\hline
\end{tabular}

aPIMs were defined based on the 2015 American Geriatric Society Beers Criteria ${ }^{\mathrm{b}}$ Fisher's exact tests or Student's t-tests were used for comparisons between the acceptance and refusal groups. The threshold for statistical significance was set at $p<0.05$

admission was $77.2 \%$. The most common categories of PIMs were benzodiazepines $(N=48,35.3 \%)$, followed by proton pump inhibitors $(N=45,33.1 \%)$, non-steroidal anti-inflammatory drugs $(N=14,10.3 \%)$ and hypnotics $(N=13,9.6 \%)$. There were no significant differences in the proportion of patients taking any PIMs at admission between the refusal group and the acceptance group (75. $9 \%$ and $78.0 \%$, respectively; $p=0.84$ ). This proportion did not differ between the two groups for each PIM category at admission.

Table 3 shows the results of binary logistic regression analysis to predict refusal of the deprescribing intervention. In both the univariable and multivariable analyses, none of the 11 variables examined were associated with the refusal of the deprescribing intervention.

\section{Discussion}

The results of this study showed that the proportion of patients taking any PIMs at admission did not differ between elderly patients refusing and accepting the deprescribing intervention. Furthermore, the number of PIMs at admission was not associated with a reduced risk of refusal for the intervention.

To our knowledge, this is the first study to evaluate the differences in the proportion of patients taking any PIMs between elderly patients with polypharmacy who accept or refuse a deprescribing intervention. Our findings indicate that elderly patients refusing deprescribing
Table 3 Summary of logistic regression results to predict deprescribing refusal

\begin{tabular}{|c|c|c|}
\hline & \multicolumn{2}{|c|}{$\begin{array}{l}\text { Odds ratio } \\
\text { (95\% confidence interval) }\end{array}$} \\
\hline & Univariable & Multivariable $^{a}$ \\
\hline Increasing age & $1.04(0.99-1.09)$ & $1.05(0.99-1.10)$ \\
\hline Male gender & $0.78(0.35-1.75)$ & $0.78(0.32-1.89)$ \\
\hline Institutional resident & $1.07(0.38-3.01)$ & $0.58(0.16-2.12)$ \\
\hline Increasing $\mathrm{CCl}^{\mathrm{b}}$ & $0.93(0.74-1.16)$ & $1.00(0.77-1.31)$ \\
\hline Dementia & $1.14(0.48-2.72)$ & $0.91(0.29-2.85)$ \\
\hline Depression & $2.20(0.72-6.75)$ & $2.05(0.58-7.23)$ \\
\hline Insomnia & $1.23(0.60-2.50)$ & $1.01(0.42-2.48)$ \\
\hline Regular drinker & $1.86(0.63-5.48)$ & $1.80(0.55-5.89)$ \\
\hline Current smoker & $3.92(0.97-15.91)$ & $3.76(0.86-16.45)$ \\
\hline $\begin{array}{l}\text { Increasing number of } \\
\text { medications at admission }\end{array}$ & $0.92(0.81-1.05)$ & $0.90(0.78-1.05)$ \\
\hline $\begin{array}{l}\text { Increasing number } \\
\text { of PIMs }^{c} \text { at admission }\end{array}$ & $1.09(0.83-1.44)$ & $1.13(0.75-1.71)$ \\
\hline
\end{tabular}

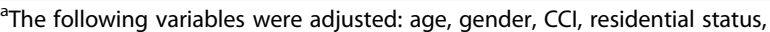
dementia, depression, insomnia, current smoker, regular drinker, number of medications at admission, and number of PIMs at admission

${ }^{\mathrm{b}}$ Continuous variable used

'PIMs were defined based on the 2015 American Geriatric Society Beers Criteria

intervention are subjected to inappropriate polypharmacy as frequently as those accepting it. Furthermore, the refusal rate for the deprescribing intervention was 39.7\%, which is consistent with past studies showing that more than $30 \%$ of patients screened for an intervention for deprescribing had declined to participate [14-16]. Given the high prevalence of polypharmacy among elderly patients, this high rate of refusal for deprescribing is disappointing. To resolve the problem of inappropriate polypharmacy among elderly patients, in addition to providing intervention for those willing to receive it, a strategy is needed for inappropriate polypharmacy among elderly patients reluctant to receive deprescribing.

Multivariable analysis was used to examine variables predicting refusal of the deprescribing intervention, but no variables were identified. Considering that factors, such as PIM use, age and CCI, could not predict willingness to deprescribe, it is possible that other uncaptured factors, such as the values and preferences of patients, are involved. Therefore, further studies are warranted to explore the association between these factors and deprescribing refusal among elderly patients with polypharmacy. In this study, the proportion of patients who took NSAIDs or opioids tended to be higher in the refusal group than in the acceptance group. Although these differences were not statistically significant, this study may be underpowered due to its small sample size. Given that the success rate of deprescribing intervention varies according to drug category in a past study [16], patients' concern that the specific medication they prefer to 
continue could be targeted for deprescribing may also affect their decision on the deprescribing intervention. Thus, the effect of specific medications also needs to be investigated in the future.

\section{Limitations}

Our results should be interpreted in the context of several limitations. First, this study utilized a retrospective design. Furthermore, this study was limited to a single center and to elderly patients admitted to the orthopedic ward who were taking five or more medications; as such, the results may not be easily generalized. Therefore, these findings should be confirmed by future prospective studies in other settings. Second, we excluded OTC drugs. Therefore, the prevalence of PIM use may be underestimated. Third, our assessment did not include potential prescription omissions [24]. Fourth, our deprescribing intervention did not target several medications, such as eye drops and topical medications. Therefore, our findings cannot be generalized to the medications excluded from this study. Fifth, the small sample size resulted in wide $95 \%$ confidence intervals for odds ratios for several variables such as smoking status, alcohol use, and depression. Therefore, the association between these variables and deprescribing refusal must be investigated in future studies with a larger sample size. Sixth, the pharmacists could not screen all consecutive orthopedic patients with polypharmacy that were seen over the study period. Therefore, the results might be affected by selection bias. Seventh, refusal to participate in a deprescribing intervention when at a hospital may differ from refusing deprescribing by a primary care physician when the patient is medically stable. Furthermore, patients who accepted the intervention may not have been willing to discontinue all their PIMs. Eighth, given that $19.1 \%$ of patients had dementia, caregiver-related factors might have affected the results. However, it should be noted that dementia was not a predictive factor for intervention refusal in the multivariable analysis. Ninth, we did not evaluate several important factors, such as the patients' knowledge of PIM or the values and preferences of the patients, which could affect their decision regarding whether to participate in the intervention. Furthermore, the selected variables in the multivariable analysis were not based on the conceptual model [11]. Finally, a tone of "research" introduced during the informed consent procedure for the deprescribing intervention may affect the rate of refusal, although the deprescribing intervention was a component of the usual care regimen in our hospital.

\section{Conclusion}

The proportion of patients taking any PIMs was not different among elderly orthopedic patients taking five or more medications who refused or accepted the deprescribing intervention. Furthermore, none of the characteristics measured were found to be associated with willingness to participate in the deprescribing intervention. Given the high prevalence of PIM use, a strategy is needed for elderly patients with polypharmacy who are reluctant to undergo deprescribing.

\section{Abbreviations \\ CCl: Charlson comorbidity index; COPD: Chronic obstructive pulmonary disease; NSAIDs: Non-steroidal anti-inflammatory drugs; PIM: Potentially inappropriate medication; SD: Standard deviation}

\section{Availability of data and materials}

Data sharing is not applicable because we did not receive informed consent for data sharing from the participants. However, the datasets generated and analyzed during the current study are available from the corresponding author upon reasonable request.

\section{Authors' contributions}

JK designed the study, analyzed the data, and wrote the main paper. KS collected the data. $\mathrm{KH}$ analyzed the data. All the authors discussed the results and implications and commented on the manuscript at all stages. All the authors have read and approved the final manuscript.

\section{Ethics approval and consent to participate}

This study was approved by the Medical Ethical Committee of National Hospital Organization Tochigi Medical Center (No. 28-20). This study was conducted in accordance with the Ethical Guidelines for Epidemiological Research in Japan. The need for individual informed consent was formally waived by the Medical Ethical Committee of National Hospital Organization Tochigi Medical Center because the data were collected from medical records and the patients were not contacted. However, according to Japanese Ethical Guidelines, we did display a poster in the waiting room of the hospital to provide information about the collection and use of data for this study and the protection of personal information.

\section{Competing interests}

The authors declare that they have no competing interests.

\section{Publisher's Note}

Springer Nature remains neutral with regard to jurisdictional claims in published maps and institutional affiliations.

\section{Author details}

'Department of Internal Medicine, National Hospital Organization Tochigi Medical Center, 1-10-37, Nakatomatsuri, Utsunomiya, Tochigi 3208580, Japan. ${ }^{2}$ Department of Pharmacy, National Hospital Organization Tochigi Medical Center, 1-10-37, Nakatomatsuri, Utsunomiya, Tochigi 3208580, Japan. ${ }^{3}$ Department of Pediatric Surgery, National Hospital Organization Tochigi Medical Center, 1-10-37, Nakatomatsuri, Utsunomiya, Tochigi 3208580, Japan.

Received: 7 July 2017 Accepted: 12 April 2018

Published online: 17 April 2018

\section{References}

1. Wolff JL, Starfield B, Anderson G. Prevalence, expenditures, and complications of multiple chronic conditions in the elderly. Arch Intern Med. 2002;162:2269-76. https://doi.org/10.1001/archinte.162.20.2269.

2. Kaufman DW, Kelly JP, Rosenberg $L$, et al. Recent patterns of medication use in the ambulatory adult population of the United States: the Slone survey. JAMA. 2002;287:337-44. https://doi.org/10.1001/jama.287.3.337.

3. Steinman MA, Landefeld CS, Rosenthal GE, et al. Polypharmacy and prescribing quality in older people. J Am Geriatr Soc. 2006;54:1516-23. https://doi.org/10.1111/j.1532-5415.2006.00889.x.

4. Lau DT, Kasper JD, Potter DEB, et al. Hospitalization and death associated with potentially inappropriate medication prescriptions among elderly nursing home residents. Arch Intern Med. 2005;165:68-74. https://doi.org/ 10.1001/archinte.165.1.68. 
5. Woodward MC. Deprescribing: achieving better health outcomes for older people through reducing medications. J Pharm Pract Res. 2003;33:323-8. https://doi.org/10.1002/jppr2003334323.

6. Reeve E, Shakib S, Hendrix I, et al. Review of deprescribing processes and development of an evidence-based, patient-centred deprescribing process. Br J Clin Pharmacol. 2014;78:738-47. https://doi.org/10.1111/bcp.12386.

7. Scott IA, Hilmer SN, Reeve E, et al. Reducing inappropriate polypharmacy: the process of deprescribing. JAMA Intern Med. 2015;175:827-34. https:// doi.org/10.1001/jamainternmed.2015.0324.

8. Tjia J, Velten SJ, Parsons C, et al. Studies to reduce unnecessary medication use in frail older adults: a systematic review. Drugs Aging. 2013;30:285-307. https://doi.org/10.1007/s40266-013-0064-1.

9. Cooper JA, Cadogan CA, Patterson SM, et al. Interventions to improve the appropriate use of polypharmacy for older people: a Cochrane systematic review. BMJ Open. 2015:5:e009235. https://doi.org/10.1136/bmjopen-2015-009235.

10. Page AT, Clifford RM, Potter K, et al. The feasibility and effect of deprescribing in older adults on mortality and health: a systematic review and meta-analysis. Br J Clin Pharmacol. 2016;82:583-623. https://doi.org/10. 1111/bcp.12975.

11. Reeve $\mathrm{E}, \mathrm{To} \mathrm{J}$, Hendrix I, et al. Patient barriers to and enablers of deprescribing: a systematic review. Drugs Aging. 2013;30:793-807. https:// doi.org/10.1007/s40266-013-0106-8.

12. Anderson K, Stowasser D, Freeman C, et al. Prescriber barriers and enablers to minimising potentially inappropriate medications in adults: a systematic review and thematic synthesis. BMJ Open. 2014;4:e006544. https://doi.org/ 10.1136/bmjopen-2014-006544.

13. Cullinan S, O'Mahony D, Fleming A, et al. A meta-synthesis of potentially inappropriate prescribing in older patients. Drugs Aging. 2014;31:631-8. https://doi.org/10.1007/s40266-014-0190-4.

14. Tannenbaum C, Martin P, Tamblyn R, et al. Reduction of inappropriate benzodiazepine prescriptions among older adults through direct patient education: the EMPOWER cluster randomized trial. JAMA Intern Med. 2014; 174:890-8. https://doi.org/10.1001/jamainternmed.2014.949.

15. Beer C, Loh PK, Peng YG, et al. A pilot randomized controlled trial of deprescribing. Ther Adv Drug Saf. 2011;2:37-43. https://doi.org/10.1177/ 2042098611400332.

16. Potter $\mathrm{K}$, Flicker L, Page A, et al. Deprescribing in frail older people: a randomised controlled trial. PLoS One. 2016;11:e0149984. https://doi.org/10. 1371/journal.pone.0149984.

17. Reeve E, Shakib S, Hendrix I, et al. Development and validation of the patients' attitudes towards deprescribing (PATD) questionnaire. Int I Clin Pharm. 2013;35:51-6. https://doi.org/10.1007/s1 1096-012-9704-5.

18. Galazzi A, Lusignani M, Chiarelli MT, et al. Attitudes towards polypharmacy and medication withdrawal among older inpatients in Italy. Int J Clin Pharm. 2016;38:454-61. https://doi.org/10.1007/s11096-016-0279-4.

19. Kalogianis MJ, Wimmer BC, Turner JP, et al. Are residents of aged care facilities willing to have their medications deprescribed? Res Social Adm Pharm. 2016;12:784-8. https://doi.org/10.1016/.jsapharm.2015.12.004.

20. Charlson ME, Pompei P, Ales KL, et al. A new method of classifying prognostic comorbidity in longitudinal studies: development and validation. J Chronic Dis. 1987:40:373-83. https://doi.org/10.1016/0021-9681(87)90171-8.

21. The American Geriatrics Society 2015 Beers Criteria Update Expert Panel. American Geriatrics Society 2015 updated beers criteria for potentially inappropriate medication use in older adults. J Am Geriatr Soc. 2015;63: 2227-46. https://doi.org/10.1111/jgs.13702.

22. Komagamine J, Miyatake S. Lack of awareness among emergency physicians of potentially inappropriate medications in elderly patients at EDs. Am J Emerg Med. 2016;34:1888-90. https://doi.org/10.1016/j.ajem.2016.06.065.

23. McMahon CG, Cahir CA, Kenny RA, et al. Inappropriate prescribing in older fallers presenting to an Irish emergency department. Age Ageing. 2014;43: 44-50. https://doi.org/10.1093/ageing/aft114.

24. O'Mahony D, O'Sullivan D, Byrne S, et al. STOPP/START criteria for potentially inappropriate prescribing in older people: version 2. Age Ageing. 2015;44: 213-8. https://doi.org/10.1093/ageing/aful145.

\section{Ready to submit your research? Choose BMC and benefit from:}

- fast, convenient online submission

- thorough peer review by experienced researchers in your field

- rapid publication on acceptance

- support for research data, including large and complex data types

- gold Open Access which fosters wider collaboration and increased citations

- maximum visibility for your research: over $100 \mathrm{M}$ website views per year

At BMC, research is always in progress.

Learn more biomedcentral.com/submissions 